\title{
Essais
}

Revue interdisciplinaire d'Humanités

$7 \mid 2015$

Normes communiquées, normes communicantes

\section{Quand la subversion nourrit la norme. La chambre d'Isabella de Jan Lauwers}

Pascale Caemerbeke

\section{(2) OpenEdition}

1 Journals

Édition électronique

URL : http://journals.openedition.org/essais/6393

DOI : $10.4000 /$ essais. 6393

ISSN : 2276-0970

Éditeur

École doctorale Montaigne Humanités

Édition imprimée

Date de publication : 1 décembre 2015

Pagination : 96-108

ISBN : 978-2-9544269-6-9

ISSN : 2417-4211

\section{Référence électronique}

Pascale Caemerbeke, "Quand la subversion nourrit la norme. La chambre d'Isabella de Jan Lauwers », Essais [En ligne], 7| 2015, mis en ligne le 26 février 2021, consulté le 28 février 2021. URL : http:// journals.openedition.org/essais/6393; DOI : https://doi.org/10.4000/essais.6393 


\section{Quand la subversion nourrit la norme. La chambre d'Isabella de Jan Lauwers}

\section{Pascale Caemerbeke}

La subversion, pensée comme «bouleversement, renversement de l'ordre établi, des idées et des valeurs reçues ", d'après Le Petit Robert, dégage un parfum de libération. Elle peut cependant, dans une double inversion, revenir au même. La subversion en art, revendiquée par certains artistes ou mis en exergue par des critiques, donne de la valeur à une ouvre et peut aussi cacher une adhésion à des normes dominantes (non marquées et donc aussi non-remarquées), sous forme ironique ou citationnelle. Il s'agit, par exemple, de redire, de faire réentendre des clichés véhiculés par des blagues ou des stéréotypes, sans vraiment y prêter attention. L'attention étant, justement, détournée par des appels subversifs qui, finalement, font consensus.

Le retournement des valeurs et des formes de penser est relatif, la subversion est toujours subversion de quelque chose à un moment et un endroit donné. Rainer Rochlitz esquisse une histoire de la subversion en art dans Subversion et subvention. "À la différence des époques prémodernes, qui soumettaient l'artiste à la censure de leurs mécènes, à la différence aussi de l'époque moderne qui faisait de l'artiste émancipé et subversif la victime d'une société largement obtuse, l'époque contemporaine tente d'institutionnaliser la révolte et de faire coexister la subversion et la subvention $»^{1}$. Dans ce contexte où la subversion devient la valeur artistique, encouragée et récupérée par le pouvoir, on peut se demander s'il s'agit encore de subversion ? Car comme l'écrit Nathalie Heinich dans L'art contemporain exposé aux rejets : "Tout se passe finalement comme si l'aide institutionnelle à l'art contemporain instaurait une sorte de "paradoxe permissif", permettant aux artistes d'être hors normes en normalisant cette transgression des normes $»^{2}$. L'auteur

1 Rainer Rochlitz, Subversion et subvention. Art contemporain et argumentation esthétique, Paris, Éditions Gallimard, 1994, p. 19.

2 Nathalie Heinich, L'art contemporain exposé aux rejets, Nîmes, Éditions Jacqueline Chambon Hachette Littératures, 1997, p. 214. 
emploie l'expression " hors normes " qui appartient à la même nébuleuse sémantique que subversion et laisse penser que l'on pourrait se déprendre des normes, alors que nous nous situons toujours par rapport à un cadre, à des valeurs, même (et peut-être même surtout) lorsque nous nous y opposons et cherchons à faire bouger ce cadre, à trouver une échappée (par le rêve, l'imagination ou l'action). Ce terme pourrait finalement définir cette position impossible d'être désigné comme subversif, c'est-à-dire remettant en question la Loi et faisant partie de la sphère des privilégiés reconnus par le pouvoir, c'est-à-dire instaurant cette Loi.

Pour penser la subversion, j'ai choisi comme exemple le spectacle de Jan Lauwers, créé au Festival d'Avignon en juillet 2004 et qui n'a cessé depuis de tourner dans le monde entier et continue de le faire en 2014. La durée de vie de ce spectacle, les prix qu'il a reçus - comme le Prix du meilleur spectacle étranger, catégorie danse, décerné par le Syndicat professionnel de la critique en France en 2005 et le Prix de l'Académie québécoise du théâtre en 2005 -, l’accueil qui lui est réservé et les articles qui ont été et sont écrits font de lui un spectacle-phare. De plus, le fait qu'il ait été reconnu avant que n'éclate la polémique d'Avignon en 2005 le place en dehors des catégories qu'a créé cette fameuse polémique. Cependant, je commencerai quand même par rappeler cette polémique pour comprendre que l'enjeu tourne autour de la «subversion" et de sa nature (esthétique, morale, politique).

\section{Avignon 2005 et la subversion en question}

Jan Fabre a été l'artiste associé de ce $59^{\mathrm{e}}$ Festival d'Avignon qui a été très fréquenté, malgré ou peut-être à cause de la polémique qu'ont déclenché certains spectacles (123000 entrées en 2005 pour 108000 entrées en 2004 et accroissement du nombre de jeunes : $20 \%$ de plus de tarifs moins de 25 ans et de tarifs étudiants par rapport à 2004). D'autre part, ce festival a généré beaucoup de débats et de mises en mots, orales et écrites, de définitions de l'art et de sa fonction dans la cité ; autrement dit, il a reposé la question de l'art et du politique. Je me focaliserai sur ce point car Jan Fabre, comme Jan Lauwers ou Wim Vandekeybus, sont flamands ; c'est-à-dire qu'ils créent dans un pays où le Vlaams Belang, parti d'extrême droite, fait entendre sa voix. Ce parti d'extrême droite, comme d'autres en Europe, met l'art contemporain sur la sellette, dénonce ses "dérives " et prône un retour à un art plus consensuel. Lors du débat qui eut lieu à la fin du festival et retranscrit dans Le cas Avignon 2005, la journaliste de Beaux-Arts Magazine parle d'une " presse poujadiste, fermée, réactionnaire ${ }^{3}$ qui serait à l'origine

3 Georges Banu et Bruno Tackels (coord.), Le cas Avignon 2005. Regards critiques, Lavérune, Éditions l'Entretemps, 2005, p. 23. 
de la polémique. Dans le même ouvrage, les sociologues Jean-Louis Fabiani, Damien Malinas et Emmanuel Ethis distinguent deux formes de critiques qui s'opposent : une critique " avant-gardiste " qui " fustige les dérives commerciales de la programmation » et une critique "plutôt conservatrice et plus institutionnelle » qui est plus " portée par la presse de droite et les médias généralistes " ${ }^{4}$. Nous voyons que le débat autour des œuvres "subversives " est aussi politique.

Dans Avignon 2005. Le conflit des héritages, Carole Talon-Hugon démontre que deux manières de penser l'art se sont affrontées : d'un côté, celle qui se réfere à une "esthétique de la réception » et juge une œuvre en fonction du plaisir esthétique qu'elle suscite; de l'autre, celle qui s'attache à une "métaphysique » de l'artiste et crédite l'œuvre en fonction des intentions et de l'originalité de son créateur. Cette démonstration très éclairante fait cependant l'impasse sur l'enjeu de ces prises de position. L'auteur écrit dans une note de bas de page : "Écartons tout de suite l'interprétation des choses en terme de conflit politique et idéologique. Bien que ce soit là une manière de contre-attaque très pratiquée. [...] Le fait que Jan Fabre ait été attaqué par l'extrême droite belge ne suffit évidemment pas à transformer le conflit en affaire politique ${ }^{5}$. Or, il me semble justement que le fait que le travail de Jan Fabre soit dénoncé par l'extrême droite oriente, qu'on le veuille ou non, la critique et rend celle-ci complexe. Le conflit entre deux attentes face à une œuvre d'art, comme un spectacle de théâtre, n'annule pas le conflit idéologique qui existe entre la défense d'un art "subversif " et le rejet de celui-ci. La difficulté pour un spectateur étant de pouvoir concilier ces approches pour se faire son opinion : juger d'un spectacle à la fois à l'aune du plaisir esthétique et du plaisir intellectuel que l'on y a pris et juger de l'aspect prétendument subversif. C'est ce dernier point qui m'intéresse.

Jan Fabre, Jan Lauwers ou Wim Vandekeybus font partie de ce que la critique française a nommé "la vague flamande $"^{6}$. Le texte est très présent chez ces trois metteurs en scène-chorégraphes; une première subversion a lieu au niveau des catégories artistiques que sont la danse, le théâtre et les arts plastiques dont sont issus Jan Fabre et Jan Lauwers. Une autre forme de subversion, d'ordre esthétique elle aussi, est liée aux corps des performeurs et à leur nudité. Toutefois, chacun de ces artistes a bien son esthétique propre et reconnaissable, je ne rentrerai pas dans ces considérations qui m’emmèneraient trop loin. Chez ces trois artistes existe une forme de subversion liée à une

$4 \quad$ Ibid., p. 47.

5 Carole Talon-Hugon, Avignon 2005. Le conflit des héritages, Du Théâtre, Hors-série n 16 , Paris, Actes Sud, 2006, p. 5.

6 Voir à ce sujet Christian Biet et Josette Féral (éd.), Théatre public, «La vague flamande : mythe ou réalité ?", n² 211, janvier-mars 2014. 
certaine morale, à des représentations admissibles ou pas, dans notre société, bref aux normes dominantes de celle-ci. Et la subversion esthétique peut parfois recouvrir des traces d'une idéologie réactionnaire. Orgie de la tolérance de Jan Fabre qui reçut le prix du jury de la critique, en 2009, dénonce la société de consommation de biens et de jouissances, avec des scènes où les corps s'exposent dans leur intimité et crée des tableaux-chocs (comme cette femme qui accouche en équilibre sur un caddie de supermarché de biens de consommation); mais il y a aussi une idéologie raciste qui, sous le prétexte d'être soumise à notre "tolérance ", est banalisée et réitérée sous formes de blagues. On retrouve cette contradiction dans Booty Looting de Wim Vandekeybus, créé en 2012, où les corps sont malmenés et poussés dans leurs limites et où des propos dévalorisants sont tenus, là encore sous forme de blagues. Le spectateur est alors tiraillé entre ce théâtre des corps qui exprime l'impossible jouissance ${ }^{7}$ et ce relâchement dans la parole qui traduit une contamination des idées d'extrême droite contre lesquels ces artistes se prononcent. Si j'ai choisi de parler plutôt du spectacle de Jan Lauwers, c'est que le spectacle se veut "réjouissant ", c'est-à-dire qu'il subvertit la subversion en défaisant l'opposition entre plaisir esthétique et sens de l'œuvre. Cependant, on retrouve cette même alchimie entre subversion liée au corps et à ses limites et contamination des idées racistes dans le langage.

\section{Jan Lauwers et la subversion de la subversion}

Jan Lauwers a étudié la peinture à l'Académie des Beaux-Arts de Gand, à une époque où " le conceptualisme dominait dans l'art » et expose à Anvers dès 1980. Un an plus tard, il monte son premier spectacle. "Je n'aimais pas le théâtre qu'alors je ne considérais pas comme un art. Cependant, il m'est apparu comme un moyen de redéfinir l'art ${ }^{8}$. Il fonde sa compagnie, Needcompany, en 1986 et tourne son premier court métrage. « Ni metteur en scène, ni peintre, ni écrivain, ni cinéaste. Je suis simplement un artiste qui tente d'utiliser tous les médiums de la création artistique. [...] Chaque médium suscite d'autres questions ". Jan Lauwers a une pensée critique sur

7 "Je situerais alors les choses ainsi : le malaise dans la jouissance, ce qui ne s'arrange pas du côté de la sexualité, des images le montrent. Je retrouve là la machine lacano-wittgensteinnienne qui m'anime sur la question de l'image, suivant la proposition du Tractatus qui énonce qu'il $\mathrm{y}$ a de l'inexprimable, qu'il y a des choses qu'on ne peut dire, et que ce qu'on ne peut dire se montre. J'en tire ici simplement que les images honteuses ne sont pas aujourd'hui à mettre au registre de la subversion et de la libération, qu'elles ne se dressent pas contre l'interdit, qu'elles font face à l'impossible, au rapport sexuel qu'il n'y a pas. ", Gérard Wajcman, "Les frontières de l'intime. Intime exposé, intime extorqué ", in Murielle Gagnebin et Julien Milly (éd.), Les images honteuses, Seyssel, Éditions Champ Vallon, 2006, p. 87.

8 Jan Lauwers, "L'art, la philosophie et le théâtre. Entretien avec Nancy Delhalle ", Alternatives théâtrales, n 85-86, Festival d'Avignon 2005, deuxième trimestre 2005, p. 40.

9 Jan Lauwers, L'énervement, Paris, Actes Sud, 2007, p. 157. 
l'histoire de l'art et s'inscrit, dès le début de sa recherche, en s'opposant au conceptualisme. "Comme artistes, nous transgressons sans cesse. L'artiste doit être à côté d'une morale, il peut poser des questions mais non proposer des choses nouvelles ${ }^{10}$. Jan Lauwers, en se démarquant du conceptualisme, se place du côté des affects.

Dans La revanche des émotions, Catherine Grenier analyse le champ artistique des débuts de la modernité jusqu'au début du XXI ${ }^{e}$ siècle pour constater l'évolution du concept par rapport à l'affect. Si la modernité artistique se développe à partir du romantisme - où l'émotion, l'expressivité, le rapport à la nature, la transcendance sont exaltés -, elle s'impose pourtant comme un anti-romantisme, dans une aspiration révolutionnaire de déconstruction. Le post-modernisme ne fera qu'exacerber cette relation de distance par rapport au monde, et l'art conceptuel domine dans les années soixante-dix. Dans les années quatre-vingt, avec la mise en question de la modernité et de la croyance au progrès - mais aussi le sida, le terrorisme, et les médias qui diffusent des images de guerre ou de Boat People - l'art se recentre sur l'individu. Dans les années quatre-vingt-dix, l'art contemporain, en faisant appel à la vidéo, à la photographie, aux installations, à la performance mais aussi de nouveau à la peinture et au dessin, réinsuffle de la narrativité et de l'affectivité, sans toutefois rompre avec le conceptuel. Enfin, depuis une dizaine d'années, le pathos fait un retour en force, sans référence théorique ou distance critique. L'artiste propose des œuvres sensibles, sans distance, pour émouvoir le spectateur, il y a une frontalité - avec un goût pour le documentaire, la peinture et la sculpture véristes, la mise en scène de mannequins, toujours la performance, la vidéo narrative, les environnements sonores ou physiques, la photographie réaliste. Il me semble que le travail de Jan Lauwers s'inscrit dans cette description d'une tendance des ouvres valorisées par la critique et sans distance critique. La subversion serait ainsi du côté du divertissement sensible, comme le revendique Jan Lauwers qui justifie son choix en l'opposant à celui de l'extrême droite en Belgique, faisant ainsi se rejoindre les extrêmes. En s'opposant au discours de l'extrême droite, autour des subventions, il revendique un art réconfortant. "L'extrêmedroite essaie actuellement de récupérer une conception du XIX ${ }^{e}$ siècle selon laquelle l'art doit être séparé du politique, donner la beauté et la consolation, le réconfort. Je suis totalement contre l'idée que l'art contemporain ne recherche plus la beauté. La Belgique connaît actuellement un grand débat suscité par le Vlaams Belang, qui propose de retirer les subventions des grands théâtres car ceux-ci ne créent plus de la beauté. La chambre d'Isabella engendre de la beauté et de la consolation. Les gens pleurent, rient. C'est une sorte de divertissement qui remplit les cœurs. Je pense que c'est très 
important. Or, je me sens très contemporain et pas du tout de droite " ${ }^{11}$. L'art "politique » d'aujourd'hui, selon Jan Lauwers, chercherait donc à reconquérir un territoire délaissé par les post-modernes, à savoir celui du divertissement émotionnel. Avant même que la polémique éclate en 2005, opposant ceux qui revendiquent leur plaisir à ceux qui font appel au sens de l'œuvre en dépit du plaisir éprouvé, Jan Lauwers subvertit ces catégories et affirme son appartenance à une idéologie " pas du tout de droite " et au divertissement. Redonnant du sens à cette forme, en en faisant un objet de revendication face à l'extrême droite. Il me semble qu'entrer dans cette dialectique entre d'un côté l'esthétique et de l'autre le politique revient à répéter la disjonction entre le fond et la forme.

La chambre d'Isabella, spectacle créé au Festival d'Avignon 2004 où il s'y est rejoué en 2009, est le premier volet d'une trilogie Sad face/Happy face, suivi par Le Bazar du homard et La Maison des cerfs. Latmosphère de La chambre d'Isabella est toute chargée de cette époque de la découverte de l'art que l'on nommait " primitif » et des avant-gardes porteuses d'une idée de liberté. On pourrait même faire une lecture critique de ce spectacle en parallèle avec une "vision " du primitivisme ${ }^{12}$. Ce spectacle est né après la mort, en 2002, du père de Jan Lauwers qui héritait de sa collection de 5800 objets, venant d'Afrique pour la plupart et collectés au cours de sa vie. Le plateau est un espace d'exposition tout blanc, avec des vitrines et une grande table couverte de ces objets.

\section{Description de La chambre d'Isabella}

J'ai vu La chambre d'Isabella le 3 juillet 2012 dans la salle bondée du Montfort-Théâtre. Je ferai une description en mon nom, ne voulant me substituer, comme beaucoup de critiques, au spectateur en général et me prémunir ainsi de généralités.

11 Jan Lauwers, "L'art, la philosophie et le théâtre. Entretien avec Nancy Delhalle ", op. cit., p. $40-41$.

12 "Primitif" a deux sens sous-entendus, contradictoires et inséparables. Par "art primitif", on a entendu d'ordinaire toute forme de création qui ne satisfait pas aux critères formels et techniques qui ont été fixés, de façon empirique, depuis la Renaissance. [...] Dans cette acception, "primitif" oscille entre la commisération et le dédain, l'humiliant et l'insultant. Depuis cette période, un deuxième emploi est apparu, dans lequel "primitif" signifie authentique, pur, naturel, par opposition à l'hypertrophie technologique des pays industriels. Le "primitif" est dans ce cas, du côté du retour aux sources, de la régénérescence par la naïveté. [...] En apparence, ces deux sens s'affrontent. Si ce n'est que le second inverse le premier sans remettre en cause la supposée "simplicité", la supposée "brutalité" du primitif proche de la terre. [...] Cette conception a soustendu les analyses du primitivisme artistique, depuis celle de Goldwater jusqu'à celle de Rubin ", Philippe Dagen, in Pierre Sterkx, Philippe Dagen et Michel Baudson (éd.), Les premiers \& les derniers, Bruxelles, Centre Wallonie-Bruxelles, 2000, p. 9. 
Jan Lauwers commence le spectacle en expliquant que son père, mort en 2002, lui a laissé une collection d'objets venant d'Afrique, dont une partie est sur le plateau. Il évoque le sarcophage d'enfant qui trônait dans sa chambre dès l'âge de six ans et le fait qu'il vivait avec ces objets, sans se poser de questions. Il présente une sculpture représentant un pénis, dit que les femmes réalisaient ce type d'objets et lance d'un air goguenard : "On voit à quoi elles pensaient! " Rires de la salle. Cette première "blague " grivoise me met mal à l'aise, ravalant les femmes qui sculptaient ces objets à des obsédées sexuelles et les objets à des godemichés. Le contact avec le public s'établit d'emblée sur cette dévalorisation. En costume blanc, il présente ensuite les performeurs-personnages : Isabella Morandi, 94 ans, aveugle, qui participe à une expérience scientifique qui lui permet de voir grâce à une petite caméra installée sur ses lunettes en projetant des images directement dans son cerveau; Alexander, l'amour de sa vie ; ses parents ; son petit-fils ; deux jeunes femmes qui jouent les deux parties du cerveau d'Isabella ; un jeune homme qui joue sa zone érogène. Puis il demande : "Tout le monde est prêt ? " Le spectacle est rythmé par l'annonce des années importantes de la vie d'Isabella. L'histoire commence en 1910, avec la naissance d'Isabella sur une île. En 1926, son père lui donne, avant de partir, une adresse à Paris. Elle s'y rend et découvre un appartement à son nom rempli d'objets et de livres, la plupart sur l'Afrique : le plateau, comme une salle de musée, où elle retrace sa vie. Isabella s'y installe, s'inscrit à la Sorbonne et devient anthropologue.

Dans la scène suivante, les performeurs viennent tour à tour présenter des objets de la chambre d'Isabella, dans l'intention de faire rire le public, en insistant sur le côté horrible ou absurde de certains objets. La musique est forte et les acteurs crient : on dirait une foire. L'ambiance est forcée.

Le vase de libation, elle s'en servait comme salière et le lourd pénis de baleine montait la garde derrière la porte, en cas de cambriolage. Elle pendait son trousseau de clés à un clou du fétiche vaudou africain. Et aussi dans le coin des sept «sait-on jamais » crucifix. Isabella était une femme pragmatique qui ne voyait de vraie signification à la beauté que quand elle était aussi utile $\mathrm{e}^{13}$.

Dans un article de 2005, intitulé "Jan Lauwers ou la subversion du théâtre ", celui-ci explique : "Je suis parti de l'obscurité de ces objets et de la fascination qu'ils exerçaient sur moi. Cela parle du siècle, mais aussi du mensonge. Tout le monde ment, surtout les chefs d'État. À travers le personnage d'Isabella, j'ai voulu parler d'une femme qui refuse de mentir $»^{14}$.

13 Jan Lauwers, La chambre d'Isabella suivi de Le Bazar du Homard, traduit du néerlandais par Monique Nagielkopf, Arles, Actes Sud, 2006, p. 17.

14 Jan Lauwers, in Guy Duplat, "Jan Lauwers ou la subversion du théâtre ", La Libre Belgique, 15 septembre 2005 [en ligne] : http://www.lalibre.be/culture/divers/article/183908/janlauwers-ou-la-subversion-du-theatre.html [consulté le 27/09/2013]. 
Effectivement, le narrateur affirme : "Jamais elle n'avait menti. Jamais $»^{15}$. Cette affirmation est déjà un mensonge. L'absence de regard critique sur l'histoire des objets, leur " fascination ", se retrouve dans cette vision simpliste du personnage d'Isabella.

En 1935, Isabella a 27 amants, nous dit-on. À l'un d'eux, Alexandre, son grand amour - jaloux parce qu'elle ne lui demande pas de quitter sa femme -, elle annonce qu'elle est enceinte, mais pas de lui. Elle accouche d'une " mulâtresse ", nous dit Alexandre, "le fruit d'une soirée arrosée et allumée dans un bordel de la place Pigalle où elle avait vu le numéro érotique d'un Noir avec un pénis en érection qui, par pure concentration et sans attouchement, arrivait à jouir. " Isabella enchaîne : "J'ai été si touchée par la vue de ce dieu noir immobile, le silence des rares spectateurs et puis brusquement la décharge du sperme, que je suis restée dans la salle jusqu'au dernier spectateur et que j'ai demandé au Noir dans la rue s'il voulait venir boire avec moi. L'homme me confia qu'il n'avait pas d'argent. Je compris que je devrais le payer et cette forme d'émancipation radicale m'excita ${ }^{16}$. Cette description de l'homme noir, assimilé à son sexe en érection, provoque des rires excités de spectateurs. Là encore, aucune distance critique dans cette description, alors qu'Isabella raconte son histoire au présent. Le point de vue de Jan Lauwers est celui d'Isabella, c'est un regard aveugle sur l'histoire, un regard blanc qui ne s'embarrasse pas de revenir sur ce passé en y adhérant. Il fait d'Isabella une femme émancipée, bousculant les tabous liés à la sexualité, mais conforme à la représentation de l'homme noir de l'époque du "Paris noir " des années trente. "Dans un contexte marqué par la montée du féminisme, c'est la séduction exercée par l'homme noir sur la femme blanche qui alimente les fantasmes, suggérant l'idée que la virilité de l'homme noir menace l'identité masculine de l'homme blanc ${ }^{17}$. Cette vision de l'homme noir comme "bête de sexe " n'est ni démentie ni déconstruite au cours du spectacle. D'ailleurs, tous le nomment Vendredi. C'est-à-dire qu'il n'a pas de nom. Isabella ne cherchera pas à revoir le père de sa fille qui restera ce "Vendredi » symbolisant la puissance sexuelle. "Finalement, la seule valeur du nègre est sa force. Sa vie n'a pas le poids inestimable d'une vie humaine. [...] Qu'il vienne d'Amérique ou d'Afrique, qu'il soit occidentalisé ou couvert de plumes, le nègre reste la bête dans l'œil de l'homme blanc ${ }^{18}$. Car si le regard de ceux qui s'intéressaient à l'art africain et à la musique jazz retournait celui véhiculé par l'imagerie coloniale, il construisait d'autres

15 Jan Lauwers, La chambre d'Isabella suivi de Le Bazar du Homard, op. cit., p. 35.

16 Ibid., p. 24.

17 Gérard Noiriel, Chocolat clown nègre. L'histoire oubliée du premier artiste noir de la scène française, Paris, Bayard, 2012, p. 180.

18 Sylvie Chalaye, Du Noir au nègre : l'image du Noir au théâtre (de Marguerite de Navarre à Jean Genet 1550-1960), Paris, L'Harmattan, 1998, p. 292. 
stéréotypes, inversés, mais tout aussi dévalorisants. "Une autre image du nègre allait s'inventer, une image qui serviraient les désirs de subversion d'une certaine avant-garde ${ }^{19}$.

Dans un mémoire de théâtre, Mélanie Dumont note une correspondance entre des personnages de Jan Lauwers et de James Joyce, elle écrit : "Celui surnommé Vendredi, archétype de l'homme noir, exhibant son pénis en érection dans un numéro érotique, que paie Isabella pour obtenir ses services sexuels, n'est-il pas justement un clin d'œil à l'un des nombreux fantasmes de Molly Bloom ? "À mon sens, ce soi-disant "archétype " de l'homme noir est juste un cliché ravalant l'homme noir à un objet sexuel. Elle souligne que : "L'humour dans ses spectacles, et tout particulièrement dans La chambre d'Isabella, repose en outre sur une complicité avec les spectateurs, des clins d'œil leur étant adressés de manière à les inclure dans la plaisanterie. [...] Par ailleurs, le sérieux de l'existence se voit également détourné par les nombreuses allusions sexuelles qui parsèment La chambre d'Isabella. Parmi les plus truculentes, il faut entre autres rappeler la description loufoque de cet homme noir en érection, capable de parvenir à la jouissance par le seul pouvoir de la concentration $»^{20}$. Effectivement, l'humour "repose sur une complicité avec les spectateurs ", une complicité basée sur une vision dévalorisante de l'homme noir et de l'Afrique comme nous allons le voir. Cette mise à distance, par l'humour, est une forme de typification, courante dans les «blagues » et qui permet, sous couvert de légèreté, de réaffirmer une séparation entre soi et ces autres, désignés comme un ensemble ${ }^{21}$.

En 1975, lorsque Franck, son petit-fils, parle de son engouement pour David Bowie et de la façon dont il danse quand il est Ziggy Stardust, elle le gifle et dit : "Tu parles comme un pédé ! "22. Là encore, la pointe homophobe passe inaperçue, comme une plaisanterie. Quand Franck lui demande :

19 Ibid., p. 318.

20 Mélanie Dumont, Lepartage des histoires dans trois spectacles de Jan Lauwers: La chambre d'sabella, Le Bazar du homard et La Poursuite du vent, Mémoire de Théâtre, Université du Québec à Montréal, 2009, p. 114-115.

21 "Sous l'effet de typifications réifiantes (concernant les femmes, les juifs, etc.), on refuse, dans un second temps, aux groupes de personnes dont il est question ces qualités personnelles qui, de manière tout à fait évidente, leur avait été attribuées du fait de l'antériorité sociale de la reconnaissance. [...] La pratique sociale qui consiste à simplement observer de façon distanciée et à saisir de manière instrumentale d'autres personnes se renforce dans la mesure où elle trouve cognitivement un soutien grâce aux typifications réifiantes, de même que ces descriptions typifiantes bénéficient d'un apport en motivations du fait qu'elles offrent à une pratique devenue unilatérale des cadres interprétatifs qui lui conviennent. De cette manière, un système de comportement se constitue qui permet que soient traités comme des "choses", des membres de groupes de personnes déterminés, parce que la reconnaissance préalable dont ils bénéficiaient leur est désormais déniée ", Axel Honneth, La réification. Petit traité de Théorie critique, traduit de l'allemand par Stéphane Haber, Paris, Éditions Gallimard, 2007, p. 116-118.

22 Jan Lauwers, La chambre d'Isabella suivi de Le Bazar du Homard, op. cit., p. 31. 
"Pourquoi n'es-tu jamais allée en Afrique ?", elle répond : "L'Afrique est ma punition ". En 1981, Franck part en Afrique occidentale, chargé de s'occuper " de la répartition de l'aide alimentaire aux divers clans " et lorsque Isabella le rejoint car il s'est pris deux balles, elle déplore : "J'en avais le souffle coupé. Aucune trace de la beauté que j'avais étudiée avec tant de soin dans ma chambre. La guerre civile avait tout détruit et les seules fleurs que j'y ai vues étaient des sacs en plastique qui flottaient au vent dans les taillis desséchés. Des chiens à demi sauvages mangeaient les pneus de la jeep $»^{23}$. L'Afrique est ravalée elle aussi à une vision stéréotypée et univoque. Dans L'Afrique, idées reçues, Hélène d'Almeida-Topor relève, entre autres clichés, " la vision globalisante du continent et de ses habitants, censés vivre, agir, penser de la même façon " ${ }^{24}$, l'Afrique vu comme "le continent de tous les malheurs ${ }^{25}$ qui vit de l'aide internationale. Nous retrouvons dans la pièce de Jan Lauwers cette vision de l'Afrique dévastatrice, car « le regard porté sur l'Afrique est plus meurtrier que ce qui s'y passe réellement $»^{26}$.

Voyons maintenant la subversion qui recouvre cette vision de l'Afrique et de l'homme noir, ainsi que la pointe homophobe. En 1975, " elle avait eu soixante-seize amants dans sa vie ${ }^{27}$, annonce le narrateur. C'est l'anniversaire de son petit-fils Franck qui a seize ans. L'acteur qui joue Franck est un jeune homme blond. Le grand-père maternel, effacé puisqu'il n'a ni nom ni histoire, est effacé symboliquement sur le plateau par le choix de l'acteur blanc. Franck et Isabella s'embrassent longuement. Derrière moi, des femmes commentent et poussent des " hou la la!" Commence alors une histoire d'amour incestueuse. "Franck me ressuscita ", dit Isabella. La subversion du tabou de l'inceste est pour ainsi dire redoublée par l'étreinte sur le plateau entre un jeune homme et une femme assez forte et de plus de soixante ans qui s'embrassent goulûment. Le narrateur annonce : " Il avait seize ans quand elle le dépucela et elle en avait soixante-neuf quand elle ressentit de nouveau la vraie jouissance comme une jeune étudiante $»^{28}$.

23 Ibid., p. 34-35.

24 Hélène d'Almeida-Topor, L'Afrique, idées reçues, Paris, Le Cavalier Bleu, 2006, p. 10.

25 «Comment se pérennise l'idée que l'Afrique est un continent plus violent que les autres? La responsabilité en incombe en grande partie au traitement de l'information par les médias. [...] Les guerres, qui constituent toujours une partie essentielle des mentions sur l'Afrique, sont encore qualifiées de "tribales", comme si cette expression suffisait à tout expliquer dans ce continent, alors qu'elle n’a jamais été employée pour des guerres civiles au Moyen Orient, dans les Balkans ou en Asie centrale. Montrer que les Africains ont, pour se battre, des intérêts autres qu'ethniques, régionalistes, ou "tribaux" sort du cadre des idées reçues et semble peu séduire les journalistes. Images et commentaires se concentrent principalement sur des aspects propres à susciter l'émotion du téléspectateur ", Hélène d'Almeida-Topor, op. cit., p. 76-77.

26 Propos de Kofi Yamgane, homme politique français d'origine togolaise, ancien secrétaire d'État, lors d'une conférence organisée par l'ONG Coordination pour l'Afrique de demain, en janvier 2003. Ibid., p. 118.

27 Jan Lauwers, La chambre d'Isabella suivi de Le Bazar du Homard, op. cit., p. 29.

28 Ibid., p. 30-31. 
La subversion de l'inceste est relevée explicitement par Rosita Boisseau dans Télérama et décrite comme "déstabilisante " : "La Chambre d'Isabella a tout d'une comédie musicale élégante et branchée dans un décor d'objets ethnographiques sidérants. Et pourtant elle met en scène une héroïne de 89 ans, aveugle, qui raconte, entre autres, comment elle est tombée amoureuse de son petit-fils». Dérangeant ? Jan Lauwers n'élude pas : "Effectivement, je mets en scène une situation inacceptable. Je ne peux pas l'imaginer dans la vie. Mais je la rends étrangement acceptable dans la pièce. Ce qui m’intéresse d'abord, c'est l'humain dans tout ce qu'il est. ${ }^{29}$.

On peut se demander pourquoi rendre cette situation amoureuse entre une grand-mère et son petit-fils acceptable ? Quel sens donner à cette subversion? N'est-elle pas totalement gratuite ? Car le tabou de l'inceste préserve l'intimité des enfants afin qu'ils se construisent comme sujets, dans la relation de pouvoir entre parents et enfants. Dans l'article du Midi Libre, la subversion de l'inceste est occultée par celle du plateau et louée comme salutaire, car l'actrice ne correspond pas aux normes corporelles de la société. "Si Isabella n'a plus 20 ans, les rides de son visage et les plis de son ventre ne la rendent pas indésirable pour Franck. Leur baiser fougueux empoigne nos tabous, décoiffe nos certitudes en ces temps de culte du corps jeune et du corps mince. Salutaire $! »^{30}$.

Lors du spectacle, tous les acteurs entonnent un chant à la gloire d'Isabella : "Elle est une femme merveilleuse ", je m'interroge sur le sens de ce merveilleux car Isabella entretient une relation sexuelle avec son petit-fils et paraît égocentrique, raciste, homophobe. Il y a une confusion entre le rôle d'Isabella et l'actrice Viviane de Muynck, qui est, sans doute, une femme formidable. «Le merveilleux » est repris dans la critique du site Evene : «Ceux qui ont vu La Chambre d'sabella à sa création [...] en parlent avec des étincelles dans les yeux. [...] Huit ans après, le sortilège agit toujours : on ressort de $\mathrm{La}$ Chambre d'Isabella en ayant pris une claque et reçu une caresse $»^{31}$.

Le spectacle est chaudement applaudi. À la sortie, je guette des spectateurs avec qui discuter. Une femme exprime sa déception, elle avait entendu une critique sur France Culture ${ }^{32}$ qui faisait l'éloge du spectacle, elle a trouvé les

29 Rosita Boisseau, "Jan Lauwers, sans concession, Le metteur en scène et plasticien bruxellois Jan Lauwers boucle sa trilogie "Sad Face / Happy Face" à Avignon. Et reste toujours aussi déstabilisant ", Télérama, n 3103, 4 juillet 2009 [en ligne] : http://www.telerama.fr/scenes/ jan-lauwers-sans-concession,44705.php [consulté le 27.09.2013].

30 Stéphanie Teillas, "Souffle de liberté dans La chambre d'Isabella ", Midi libre, 30 juin 2011 [en ligne] : http://www.midilibre.fr/2011/06/29/souffle-de-liberte-dans-la-chambre-disabella,345921.php [consulté le 27/09/2013].

31 Critique d'Étienne Sorin, paru sur le site Evene [en ligne] : http://www.evene.fr/culture/ agenda/la-chambre-d-isabella-6875.php?critiques [consulté le 27/09/2013].

32 Émission de La Grande Table, du 19 juillet 2012 où Caroline Broué s'entretient avec Viviane de Muynck. 
chants, la musique et les danses très jolis mais le spectacle ne l'a pas émue et lui a semblé superficiel.

Effectivement, tout dans ce spectacle est attrayant, même le frisson de la subversion, mais il n'y a aucun regard critique, ni sur ces objets, ni sur le colonialisme. Jan Lauwers s'est attaché à la forme du spectacle, comme il en est resté à la forme des objets : la musique jouée en direct (Jan Lauwers joue aussi de la guitare électrique), les chants - comme "We are the people. The people who never stop. We just go on and on and on" repris par tous les interprètes en début et à la fin du spectacle qui laisse flotter sa mélodie dans la tête du spectateur -, les moments de danse sensuels et énergiques, la scénographie qui transforme le plateau en espace d'exposition, l'emploi de la vidéo (Jan Lauwers manipule une caméra et filme des objets qui apparaissent sur un écran), la simultanéité des actions. Tous ces éléments donnent un éclat au spectacle, on pourrait presque dire un clinquant (d'ailleurs le plateau est éclatant de blancheur, tout comme le costume de Jan Lauwers et d'Alexandre) qui dissout toute ombre et toute contradiction.

\section{Conclusion}

Avec cet exemple, nous voyons comment la soi-disant subversion peut masquer des représentations qui ne font que réaffirmer une logique de domination, dont ne font état aucun des articles ou des commentaires. Des stéréotypes, emballés dans une subversion superficielle, ne sont ni questionnés ni remis en question. Et la critique reste à la surface, aveuglée par la prétendue subversion qui n'est que poudre aux yeux et fait consensus. En revenant aux propos de Jan Lauwers, on comprend que ce spectacle, et sa prétendue subversion, s'adresse à, comme il le dit, une " élite ». "Si je fais du théâtre, c'est parce que c'est complètement inutile dans cette société-là. [...] Le théâtre est cher, inutile et ne touche pas les masses - il est très élitaire -, cela le rend irrécupérable ${ }^{33}$. Dans cette optique, le théâtre se réduit à un divertissement qui éloigne de toute pensée critique et réellement subversive. Par contre, « le pouvoir performatif de la parole qui convoque non seulement les mots mais aussi les actions du passé et les fait revivre au présent " ${ }^{34}$ a dans ce spectacle un réel pouvoir subversif, puisqu'il transporte une idéologie

33 Jan Lauwers, "L'art, la philosophie et le théâtre. Entretien avec Nancy Delhalle », op. cit., p. 20.

34 "La parole y est assumée à la fois comme artifice et comme principale action : dire suffit à faire exister, la narration ouvrant un autre espace, celui de l'imaginaire de chaque spectateur devenu complice de la création de l'histoire qui se raconte devant lui. Lauwers crée ainsi une communauté autour des personnages présents sur scène et autour de l'histoire qui se raconte au présent entre les comédiens et les spectateurs, histoire qui n'existe pas encore mais se crée à-même le plateau ", Gabrielle Girot, “"Que peut-on raconter aujourd'hui au théâtre ?", Sad Face/Happy Face de la Needcompany ", Théatre public, op. cit., p. 64. 
raciste, dénoncée par Jan Lauwers, dont aucun des critiques ne fait état. Comme si l'extrême droite avait imposé son vocabulaire à ceux qui pourtant s'y opposent.

\author{
Pascale Caemerbeke \\ Université Paris 3-Sorbonne nouvelle \\ caemerbeke@hotmail.com
}

\begin{abstract}
Résumé
Que veut-on transmettre lorsqu'on utilise les termes " hors-norme " ou "subversion " ? La subversion affichée peut, dans certains cas, réitérer des clichés sans qu'ils soient relevés. En nous penchant sur le spectacle de Jan Lauwers, La chambre d'Isabella - en tournée depuis neuf ans et loué par les critiques pour son caractère subversif - nous verrons que l'élément subversif, lié à la sexualité, fait accepter une vision de l'homme noir et de l'Afrique dévalorisante.
\end{abstract}

\title{
Mots-clés
}

Normes, hors-normes, subversion, clichés, La chambre d'Isabella de Jan Lauwers.

\begin{abstract}
What do we want to signify when we use the terms "outside the norm " or "subversion"? The exhibited subversion can, at the same time, repeat stereotypes. We will take a look at $\mathrm{La}$ chambre d'Isabella by Jan Lauwers - a danse performance played around the world during nine years and highly acclaimed by critics for its subversive potential- to discover that the subversion related to sexuality is hiding a denigrating image of black men and Africa.
\end{abstract}

\section{Keywords}

Social norms, outside the norm, subversion, stereotypes, La chambre d'Isabella by Jan Lauwers. 